\title{
Reliability of MYZONE® MZ-3 Heart Rate Monitor Integrated into a Video-Consulting ApplicationA Study for the Future of Tele-prehabilitation for patients prior to major cancer surgery
}

\author{
Jamie L. Waterland ${ }^{1 *}$, Luis Cuadros ${ }^{2}$, Hilmy Ismail ${ }^{1}$, Yesim Karabiyik ${ }^{1}$, Kwok M Ho ${ }^{3}$ and Bernhard Riedel ${ }^{1}$
}

${ }^{1}$ Peter MacCallum Cancer Centre, Australia

${ }^{2}$ Monash Health, Melbourne, Australia

${ }^{3}$ School of Veterinary \& Life Sciences, Murdoch University, Australia

Submission: January 23, 2020; Published: February 10, 2020

*Corresponding author: Jamie L. Waterland, Peter MacCallum Cancer Centre, 305 Grattan Street, Melbourne, VIC 3000, Australia

\begin{abstract}
Background: Prehabilitation in patients undergoing major cancer surgery has the potential to decrease major perioperative complications. However geographical barriers can make it access difficult. Modern technological advances in telecommunications and wireless-enabled wearable activity trackers, may allow health services to deliver innovative programs to those who remain geographically isolated.

Introduction: We conducted a proof of concept study to evaluate the reliability of a commercially available heart rate monitor, the MYZONE® MZ-3, and integrated it into a custom developed video-consulting application designed to deliver prescriptive exercise training to patients undergoing major cancer surgery.

Materials and Methods: Paired heart rate data from each participant was captured and analyzed using a Bland-Altman plot. In addition, patient experience of the technology was collected by way of questionnaires.

Results: 20 participants were consented for the study and underwent a standardized Cardiopulmonary Exercise Testing (CPET) protocol A total of 1,674 paired heart rate data points were analyzed. The bias (mean difference) between the Vyntus CPET ECG and the video-consulting application integrated MYZONE® MZ-3 heart rate monitor was 0.5 beats per minute with an adjusted $95 \%$ limits of agreement of -7.5 to 8.2 beats per minute.

Conclusions: In our study we found that our custom video-consulting application provided reliable heart rate monitoring that could be used to deliver tele-prehabilitative services. The overall experiences of using the application by the study participants were rated as good or excellent. This study further endorses a technology that can reliably deliver much needed and welcomed services in the field of perioperative medicine.

Keywords: Heart rate monitor; Prehabilitation; Remote consultation; Telemedicine; Tele-prehabilitation.
\end{abstract}

\section{Introduction}

Prehabilitation is a process on the continuum of care thatoccurs between the time of cancer diagnosis and the beginning of acute treatment (e.g. surgery). Prehabilitation includes assessments of baseline level of fitness and provision of targeted interventions to improve fitness, mitigate risk and reduce the incidence and the severity of current and future impairments [1]. Multiple studies have demonstrated the benefits of prehabilitation in improving physical function [2,3] and decreasing major complications after surgery by almost $50 \%$ [4-7]. Clinical guidelines for pre-operative exercise training in patients awaiting major non-cardiac surgery advocate for supervised exercise programs with therapists with relevant expertise [4]. Attendance at multiple supervised, structured, responsive exercise sessions is a commonly reported 
barrier to prehabilitation by patients [5]. Home-based exercise interventions, although preferred by many patients, may be suboptimal due to the lack of supervision and appropriate exercise progression [6]. Published data from our group indicate that these access barriers may exist for a significant group of patients [7]. Contributing causes include inaccessibility to inhospital supervised exercise programs [8]. Tele-prehabilitation, using well designed smart technology may be an opportunity to overcome these barriers $[9,10]$. Remote heart rate (HR) monitoring during exercise allows for real-time control of exercise intensity and training effect [11]. A number of heart rate monitors (HRM) have been evaluated for accuracy and precision against the electrocardiogram (ECG) with varying results [11].

The MYZONE $^{\circledR}$ MZ-3 HR monitor is a popular fitness device that is also priced at a level that would be affordable to a hospital prehabilitation service. It records and transmits HRs at a frequency of $1 \mathrm{~Hz}$ to a Bluetooth-enabled device with the MYZONE $^{\circledR}$ application installed. We have previously studied the accuracy and precision of the MYZONE ${ }^{\circledR}$ heart rate monitor as a stand-alone device in a non-clinical setting with healthy volunteers and have found the bias between ECG and the MYZONE ${ }^{\circledR}$ MZ-3 was 0.4 beats per minute (bpm). Adjusted 95\% LOA were found to be -4.5 to $5.3 \mathrm{bpm}$. The percentage error (95\% LOA/mean HR) was $3.6 \%$. This device was found to have an acceptable level of reliability for use in healthy volunteers performing exercise across a wide range of HRs [12]. This is the first study to the authors' knowledge to validate a telehealthcapable application for intervention in a preoperative oncology population. The use of a validated HR monitor coupled with visual and audio feedback of the patient during exercise may present a unique opportunity for monitored targeted exercise programs within the community setting and overcome geographical barriers to access supervised prehabilitation exercise programs. A custom-built telecommunications application was designed to provide audio and visual communication between a patient and clinician over an Internet connected device. In addition, the MYZONE ${ }^{\circledR}$ MZ-3 heart rate monitor has been integrated into the application to stream the patient's heart rate to the clinician in real time. During our pilot study of this technology, the application was hosted on a dedicated web server configured on a Linux based (Debian 8.0 64-bit) operating system with public access to the internet. The clinician accessed the application portal via a URL link on any Google Chrome internet browser. The patient accessed the portal via an android-based application designed to run on mobile devices. The application allowed audio-visual connectivity with the clinician in three easy steps. Once a secure connection with the portal was established, clinicians were able to initiate a session with a patient in a virtual waiting room. The session was then terminated by the clinician or patient upon completion of the exercise program.

\section{Objective}

We set out to assess the clinical utility of a telecommunications application ("Application") incorporating a validated heart rate monitor for delivery of tele-prehabilitation.

\section{Primary Objective}

To assess the reliability of the MYZONE ${ }^{\circledR}$ MZ-3 HR monitor incorporated into our Application as compared to a Class II, FDAapproved ECG monitor (Vmax Vyntus ECG, Centrisuite V 2.9) during routine cardiopulmonary exercise testing (CPET) in cancer patients presenting for major cancer surgery.

\section{Secondary Objectives}

To assess ease of set up and ease of use of the Application for patients with limited technical knowledge. To assess the user experience of using the Application in terms of the following domains: sound quality, ease of use, likelihood to use again and potential comfort using the Application within the home or community-based setting.

\section{Materials and Methods}

This study was conducted after approval by the Peter MacCallum Cancer Centre low risk ethics committee (Ref no. LNR/17/PMCC/50). Adults >18 years of age, English speaking or with the availability of an accredited interpreter and scheduled to have CPET as a routine test prior to major Cancer Surgery were approached for inclusion. Patients were excluded if they were: unable or unsuitable to complete CPET, had a history or a cognitive impairment or had an active pacemaker or an automated implantable cardioverter-defibrillator (AICD). The MYZONE ${ }^{\circledR}$ MZ-3 HR sensor was attached by a chest strap to the trial participant during CPET and the HR signal was captured and recorded to a file stored locally on the mobile device (Motorola Moto G $3^{\text {rd }}$ Generation with Android Version 6.0.1). ECG leads were secured to the subject during CPET. Transmission of the ECG signal was via wireless technology to the Vyntus Centrisuite Software V 2.9. The participant was then instructed to initiate a session via the Application. Upon initiation of a session with a clinician in an adjacent room, the patient was then instructed to complete formal CPET. The ECG derived HR was accessed using Centrisuite's reporting software. Following the CPET session, participants were surveyed on aspects of their experience using the Application. The survey aimed to capture patient feelings regarding the Application including sound quality, ease of set up, preference to use telehealth again as well as comfort using 
telehealth such as the application to allow supervised exercise in the home environment.

\section{Data Collection}

The Vyntus CPET software recorded HR information at fivesecond intervals from the start of the CPET session. The Application recorded HR information at one-second intervals. In our previous study [12], system clocks on both devices were synchronized to allow accurate pairing of the HR data set. A limitation of the Vyntus software was that no system time information was recorded along with the captured HR. In order to ensure correct pairing of the HR data captured on both systems, the peak or maximum heart rate recorded on the Vyntus software was cross-referenced with the peak HR recorded on the Application. Once this point was identified, the remaining paired HR data was extrapolated in 5-second increments on either side of the identified peak HR. Unpaired HRs were excluded from our analysis. See Figure 1 for an example of HR trends following this technique for pairing HR data in a single participant.

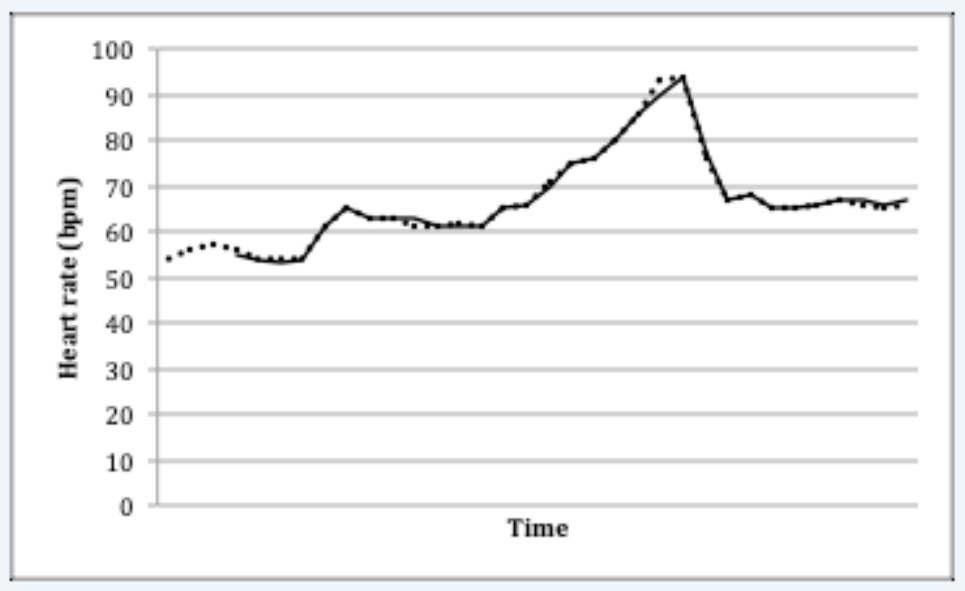

Figure 1: Vyntus heart rate (dotted) and Application heart rate (solid) following synchronization of paired data points for a single participant.

\section{Statistical Analysis}

The agreement between the heart rates generated by Vyntus software and the Application was analyzed using a Bland-Altman plot. To account for several paired measurements for each participant, the analysis was adjusted to repeated observations derived from the same participants [12]. The bias (mean difference), standard deviation (SD), 95\% limits of agreement (LOA) allowing for repeated measures derived from the same individuals, and plot were derived using Medcalc Statistical Software version 18.11.3 (MedCalc Software bvba, Ostend, Belgium; https://www.medcalc.org; 2019). On a number of occasions, the Vyntus software (gold standard) heart rate recording was erroneous and clearly artifactual and thus could not be paired to the corresponding application data points (Figure 2). It is our assumption that during these periods, there was a brief loss of contact between the wireless Vyntus ECG signal generator and receiver. These outliers were excluded from statistical analysis, as they were not in keeping with the overall trend. We acknowledge these corrections as an adjustment to the data acquisition and consider it unlikely to have any clinically significant effects on our results.

\section{Results}

Twenty-seven patients were approached for inclusion into the study with 20 (74\%) consenting to participate. Three declined to participate, three were unable to complete testing (1 with distress, 2 with to musculoskeletal issues and pain and one due to equipment issues) (Table 1). A total of 20 patients having CPET were included in the study. Mean age of the participants was 62.6 years. There were 10 female and 10 male participants. Data from 4 patients were excluded due to incomplete data acquisition or unexpectedly large discrepancies in the HR data. From the remaining 16 patients, 55 data points from a total of 1829 data points remained unpaired and were excluded from analysis. Furthermore, 100-matched HRs from two patients were excluded from analysis due to clear artifact on the Vyntus software (Figure 2). We believe this is justified, as there was a clear discrepancy in the HR trend during exercise. The total number of remaining 
paired HR data points analysed was 1674 from 16 participants. The results (Figures 3,4) show that the bias between ECG and the Application was 0.5 beats per minute (bpm). Adjusted $95 \%$ LOA were found to be -7.5 and $+8.2 \mathrm{bpm}$. The percentage error (95\% LOA/mean HR) was 15.5\%. The Concordance Correlation Coefficient between the Application and Vyntus HR measurements was 0.98 (0.987 to 0.989). Thirteen of the included participants (65\%) completed the survey after CPET testing with the reasons for non-completion being conflicting clinical appointments or transport logistics. Analysis of the subjective acceptability of the application revealed that 12 out of the 13 participants surveyed would use telehealth again. Twelve of the participants also agreed that they would feel comfortable using a similar device at home or in a gym close to home whilst exercising. Two participants reported issues setting up or hearing the therapist during CPET, however both reported a history of hearing impairment. Despite this $77 \%$ of participants rated the overall experience of using the application as good or excellent.

Table 1: Patient Demographics.

\begin{tabular}{|c|c|}
\hline Characteristic & Total sample (n=20) \\
\hline Male, n (\%) & $10(50 \%)$ \\
\hline Age (years), Mean (SD) \\
Range & $62.6(9.0)$ \\
\hline Cancer Diagnosis, n (\%) & $73-73$ \\
Pseudomyxoma peritonei & $7(35)$ \\
Oesophageal & $6(30)$ \\
Colorectal & $5(25)$ \\
Prostate & $1(5)$ \\
Haematological & $1(5)$ \\
\hline Comorbidities, Mean & 2.2 \\
Range & $0-6$ \\
Hypertension, n (\%) & $7(35)$ \\
Asthma, n (\%) & $3(15)$ \\
History of Stroke, n (\%) & $3(15)$ \\
Heart disease, $n$ (\%) & $2(10)$ \\
Type II diabetes mellitus, n (\%) & $2(10)$ \\
Malnutrition, n (\%) & $1(5)$ \\
Obesity, n (\%) & $1(5)$ \\
\hline Chronic obstructive airway disease, $n(\%)$ & $1(5)$ \\
\hline Residential location, n (\%) & $15(75)$ \\
Metropolitan Melbourne & $4(25)$ \\
\hline Tasmania & $4(20)$ \\
\hline Northern Territory & $1(2.5)$ \\
\hline
\end{tabular}

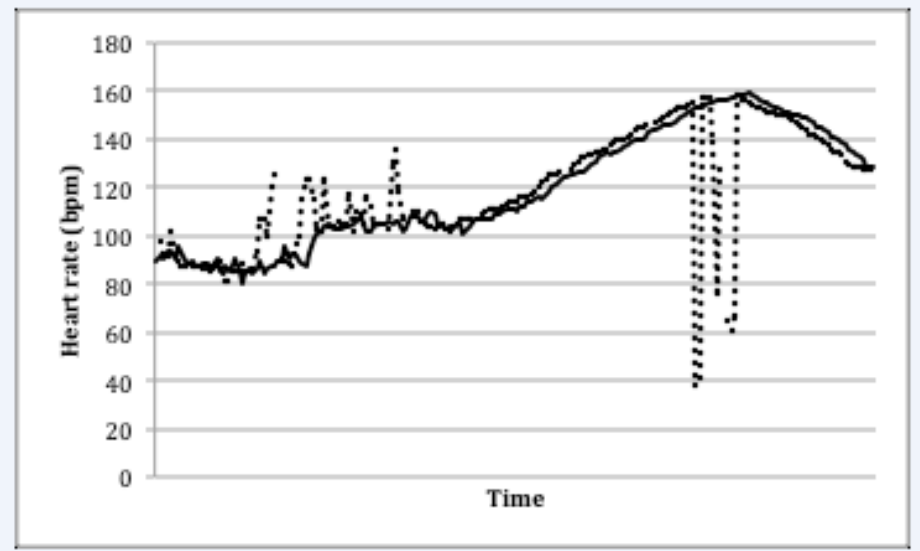

Figure 2: Vyntus heart rate (dotted) and the Application heart rate (solid). Suspected artifact heart rates recorded by the Vyntus software not in keeping with the overall trend. 


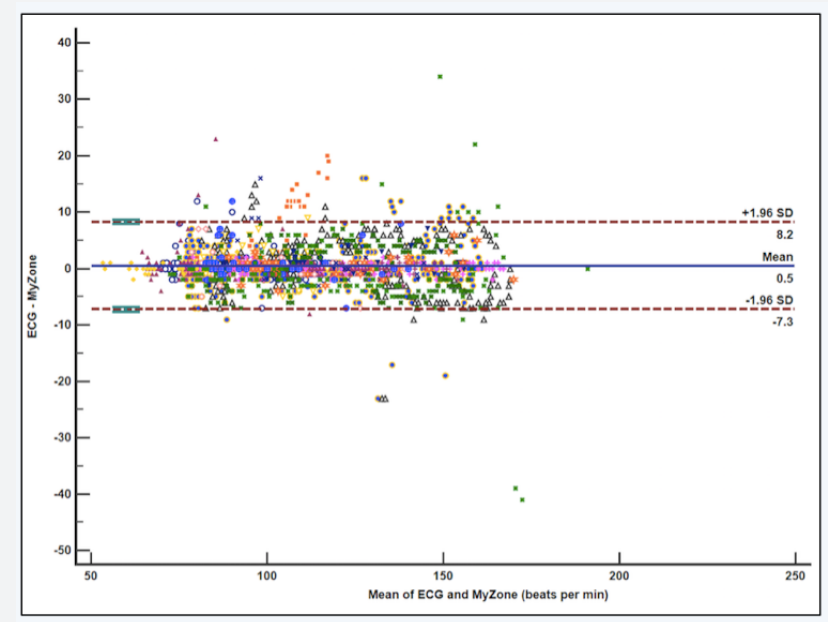

Figure 3: Bland Altman plot for Application heart rate and Vyntus ECG.

\begin{tabular}{|l|c|}
\hline \multicolumn{2}{|c|}{ Agreement with ECG } \\
\hline Device & Concordance Correlation Coefficients $(95 \% \mathrm{CI})$ \\
\hline Polar H7* & $0.99(0.987-0.991)$ \\
\hline Apple Watch* & $0.91(0.884-0.929)$ \\
\hline Fitbit Charge HR* & $0.84(0.791-0.872)$ \\
\hline MyZone HRM & $0.98(0.987$ to 0.989) \\
\hline
\end{tabular}

Figure 4: Comparison of Concordance Correlation Coefficients across commercially used HRMs* [12].

\section{Conclusion}

This pilot version of a specifically designed Application with an integrated commercially available HR monitor (MYZONE ${ }^{\circledR}$ MZ3) to deliver tele-prehabilitation services has an acceptable level of reliability and user experience for use in patients performing exercise across a wide range of Heart Rates. When compared to previously published analysis on the accuracy of three commonly used commercial wrist worn HRMs, the MYZONE ${ }^{\circledR}$ HRM had a Concordance Correlation Coefficient superior to the Apple watch and Fitbit Charge HR monitors and was found to be comparable to the Polar H7 HRM (Figure 4). Our Application may have the potential for delivering tele-prehabilitation to patients undergoing major cancer surgery and the accuracy of heart rate capture is superior to commercially available wrist worn HRMs. This is of particular importance to patients presenting for cancer surgery who may have significant cardiac pathology, necessitating accurate and reliable HR monitoring during exercise prehabilitation. Prehabilitation services can use these devices to prescribe and monitor exercise progress and improvements that we hope will have a meaningful impact on recovery from major surgery. Future work needs to be done to improve the design and robustness of a telecommunications application capable of delivering telehealth services to the elderly population presenting for non-cardiac major surgery. This model of care can be expanded to post-operative rehabilitative services as well as non-surgical patient care.

\section{Author Disclosure Statement}

The Authors declare that there is no conflict of interest.

\section{References}

1. Silver JK, J Baima (2013) Cancer prehabilitation: an opportunity to decrease treatment-related morbidity, increase cancer treatment options, and improve physical and psychological health outcomes. Am J Phys Med Rehabil 92(8): 715-727. 
2. West MA, Loughney L, Lythgoe D, Barben CP, Sripadam R, et al. (2015) Effect of prehabilitation on objectively measured physical fitness after neoadjuvant treatment in preoperative rectal cancer patients: a blinded interventional pilot study. British Journal of Anaesthesia 114(2): 244-251.

3. Carli F, Charlebois P, Stein B, Feldman L, Zavorsky G, et al. (2010) Randomized clinical trial of prehabilition in colorectal surgery. British Journal of Surgery 97(8): 1187-1197.

4. Tew GA, Ayyash R, Durrand J, Danjoux GR, et al. (2018) Clinica guideline and recommendations on pre-operative exercise training in patients awaiting major non-cardiac surgery. Anaesthesia 73(6): 750 768.

5. Ferreira V, Agnihotram RV, Bergdahl A, van Rooijen SJ, Awasthi R, et al (2018) Maximizing patient adherence to prehabilitation: what do the patients say? Supportive Care in Cancer 26(8): 2717-2723.

6. Ormel HL, van der Schoot GGF, Sluiter WJ, Jalving M, Gietema JA, et al. (2017) Predictors of adherence to exercise interventions during and after cancer treatment: A systemativ review. Psycho-Oncology 27(3): 713-724.

7. Huang GH, Ismail H, Murnane A, Kim P, Riedel B, et al. (2016)
Structured exercise program prior to major cancer surgery improves cardiopulmonary fitness: a retrospective cohort study. Supportive Care in Cancer 24(5): 2277-2285.

8. Ismail H, Prue Cormie, Kate Burbury, Jamie Water, Linda Denehy, Bernhard Riedel, et al. (2018) Prehabilitation Prior to Major Cancer Surgery: Training for Surgery to Optimize Physiologic Reserve to Reduce Postoperative Complications. Current Anesthesiology Reports 8(4): 375-385.

9. Varnfield M, Karunanithi M, Lee CK, Honeyman E, Arnold D, et al. (2014) Smartphone-based home care model improved use of cardiac rehabilitaiton in postmyocardial infarction patients: results from a randomised controlled trial. Heart 100(22): 1770-1779.

10. Sabesan S, J Kelly (2015) Implementing tele health as core business in health services. MJA 202(5): 231-233.

11. Cuadros L, H Ismail, K Ho (2017) Evaluation of Reliability of MYZONE MZ-3 Heart Rate Monitor: A Study for the Future of Telephysiotherapy for Preoperative Prehabilitation in Cancer Patients. Telemedicine \& E-Health 23(4): 334-338.

12. Robert Wang, Gordon Blackburn, Milind Desai, et al. (2017) Accuracy of Wrist-Worn Heart Rate Monitors. JAMA Cardiol 2(1): 104-106.

\section{Your next submission with Juniper Publishers} will reach you the below assets

- Quality Editorial service

- Swift Peer Review

- Reprints availability

- E-prints Service

- Manuscript Podcast for convenient understanding

- Global attainment for your research

- Manuscript accessibility in different formats

( Pdf, E-pub, Full Text, Audio)

- Unceasing customer service

Track the below URL for one-step submission https://juniperpublishers.com/online-submission.php 